\title{
Added value of circulating miRNA expression profiling to sonographic TI-RADS classification in the diagnosis of thyroid nodules
}

\author{
YU MAO, FENGJIAO ZHANG, LI HE, FANG LUO, \\ LEI LI, YAJIE HUO and ZHIQIANG KANG \\ Department of Endocrinology, Zhengzhou Central Hospital Affiliated to \\ Zhengzhou University, Zhengzhou, Henan 450007, P.R. China \\ Received June 26, 2019; Accepted February 12, 2020
}

DOI: $10.3892 /$ etm.2020.8870

\begin{abstract}
Potential use of sonographic TI-RADS classification combined with circulating miRNA expression profiling in the diagnosis of thyroid nodules was explored. Retrospective analysis was performed on clinical data of 121 patients with thyroid nodules. The biopsy specimens of patients obtained through ultrasound-guided aspiration and blood specimens were evaluated in Zhengzhou Central Hospital Affiliated to Zhengzhou University from June 2018 to June 2019. In addition, the blood specimen test results of 121 healthy volunteers (control group) who underwent physical examination were retrospectively analyzed. Results of sonographic TI-RADS classification and circulating miRNA expression profiling were compared with the pathological results. Of the 212 nodules, 2 fell into TI-RADS category 2 and were diagnosed as benign. Malignant nodules accounted for $4.35,37.14,84.78,93.33$ and $96.77 \%$ of those nodules that fell into TI-RADS categories 3, 4a, 4b, 4c and 5, respectively. Of the 121 patients, $92.55 \%$ had with nodular goiter, $3.31 \%$ had inflammatory nodules, $2.48 \%$ toxic nodular goiter, $0.83 \%$ thyroid cysts and $0.83 \%$ thyroid tumors. A nodule that fell into a higher TI-RADS classification category had a higher risk of malignancy. The expression levels of miRNA146b, miRNA187, miRNA375, miRNA-222-3p and miRNA-151a-5p were higher, while the level of miRNA138 was lower, in patients with either benign or malignant thyroid nodules compaed to those in the control group. The expression levels of miRNA146b, miRNA187, miRNA375, miRNA-222-3p and miRNA-151a-5p were higher, while the
\end{abstract}

Correspondence to: Dr Zhiqiang Kang, Department of Endocrinology, Zhengzhou Central Hospital Affiliated to Zhengzhou University, 195 Tongbai Road, Zhengzhou, Henan 450007, P.R. China

E-mail: k8b9gw@163.com

Key words: thyroid nodules, sonographic TI-RADS classification, circulating miRNA expression profiling, diagnosis level of miRNA138 was lower, in patients with malignant thyroid nodules than those in patients with benign thyroid nodule $(\mathrm{P}<0.05)$. The AUC of the combined diagnostic method was 0.973 , which was significantly different from the AUCs of the individual diagnostic method $(\mathrm{P}<0.05)$. In conclusion, sonographic TI-RADS classification combined with circulating miRNA expression profiling can improve the diagnosis of thyroid nodules.

\section{Introduction}

Thyroid nodules are common thyroid lesions, with increasing incident in recent years. Approximately 5-10\% of thyroid nodules are cancerous (1). Treatments and prognosis of patients with benign and malignant thyroid nodules are quite different. In order to reduce unnecessary physical, mental and economic burdens to patients, clinical diagnostic differentiation of benign and malignant thyroid nodules is of great significance (2). At present, clinical diagnosis of thyroid nodules mainly depends on radioisotope scan, ultrasound, MRI and CT. Ultrasound is the clinically preferred imaging method due to its convenience and non-invasiveness. However, clinicians may have subjective judgement in interpreting the ultrasound image, which may affect the diagnosis (3). In order to avoid diagnostic deviations due to subjective judgements, American College of Radiology (ACR) divided Thyroid Imaging Reporting and Data System (TI-RADS) into 5 categories, making clinical diagnosis more standardized (4). MicroRNAs (miRNAs) have been shown to be involved in several cellular processes such as cell growth, differentiation, apoptosis and adhesion. It was reported that miRNAs are abnormally expressed in cancers such as lung cancer, breast cancer and thyroid cancer. In addition, the miRNA expression levels vary in different types of thyroid diseases (5). Therefore, miRNA expression profiling can help differentiate benign and malignant thyroid nodules. In order to improve diagnostic accuracy of thyroid nodules, in this study the diagnostic power of the combination of sonographic TI-RADS classification and circulating miRNA expression profiling was investigated. 


\section{Patients and methods}

Patients. Retrospective analysis was performed on clinical data of 121 patients with thyroid nodules. Their biopsy specimens obtained through ultrasound-guided aspiration and blood specimens were evaluated in Zhengzhou Central Hospital Affiliated to Zhengzhou University (Zhengzhou, China) from June 2018 to June 2019. In addition, the blood specimen test results of 121 healthy volunteers (control group) who underwent physical examination in Zhengzhou Central Hospital Affiliated to Zhengzhou University during the same period of time were retrospectively analyzed. Of the 121 patients, there were 73 males and 48 females, aged $18-75$ years with a mean age of $(43.29 \pm 6.49)$. Nodule diameters were in the range of $3-51 \mathrm{~mm}$ with a mean diameter of $(15.95 \pm 9.73) \mathrm{mm}$. The control group included 121 healthy volunteers, 68 males and 53 females, aged 19-77 years with an average age of $45.21 \pm 6.36$.

Patients who met following criteria were recruited into the study: i) age no younger than 18 years; ii) no thyroid surgery history; iii) no history of using anti-thyroid drugs or other drugs that affect thyroid function; iv) no history of having other malignancies; and v) with complete clinical and follow-up data. patients who met following criteria were excluded from the study: i) diagnosed with other conditions such as acute or chronic diseases or diseases of the immune system; ii) with severe disorders associated with heart, liver, kidney and lung; iii) with solitary nodule; and iv) with incomplete clinical data such as not receiving ultrasound exam and/or circulating miRNA tests.

The study was approved by the Ethics Committee of Zhengzhou Central Hospital Affiliated to Zhengzhou University. Signed informed consents were obtained from the patients and/or the guardians.

\section{Methods}

Sonographic TI-RADS classification. All examinations were performed in a blinded manner. Ultrasound examinations were performed on all subjects using a Philips IU Elite color Doppler ultrasound system at a probe frequency of $7.5-12 \mathrm{MHz}$ and a mechanical index of 0.05-0.06. According to the 5 categories of TI-RADS provided by Kwak et al (6), nodules, hypoechoic or very low echo, irregular boundaries, microcalcification, vertical and horizontal $>1$, were the five ultrasound image characteristics used as the criteria for the evaluation of malignant thyroid nodules. The classification was as follows: TI-RADS 1, normal thyroid; TI-RADS 2, benign lesion; TI-RADS 3, no such malignant features; TI-RADS $4 \mathrm{a}$, one of the above malignant features; TI-RADS $4 \mathrm{~b}$, two of the above malignant features; TI-RADS $4 \mathrm{c}$, three or four of the above malignant features; TI-RADS 5, five of the above malignant features. All nodules were diagnosed by two sonographers who had experience in evaluating the degree of benign and malignant thyroid nodules using TI-RADS. When their opinions were different, the thyroid fine needle puncture was further performed.

Ultrasound-guided fine needle aspiration biopsy. For the accuracy of the results and the judgement of the diagnosis, all the patients underwent FNA. Biopsy specimens of the thyroid nodules were obtained through two ultrasound-guided fine needle aspirations performed by experienced color Doppler sonographers. The first aspiration was performed using a 7-gauge needle under negative pressure. The obtained tissue was smeared on slides. The second aspiration was performed using a CL-type biopsy needle (23Gx50 mm, Hakko Corp.). The obtained tissue was stored in formalin solution. Cells were obtained and smeared on slides after culture in media. The slides were examined under a microscope to determine the pathological characteristics of the nodules.

Circulating miRNA expression profiling. Peripheral blood samples were collected from all subjects. About $5 \mathrm{ml}$ of peripheral venous blood was taken from the elbow using an EDTA anticoagulant tube (before surgery for patients with thyroid nodules). The whole blood samples were subjected to immediate centrifugation at $1,000 \mathrm{x} \mathrm{g}$ and $4^{\circ} \mathrm{C}$ for $10 \mathrm{~min}$. Plasma obtained from the supernatant was dispensed in $300-\mu 1$ aliquots into cryotubes and stored at $-80^{\circ} \mathrm{C}$. Circulating RNAs were extracted through multiple steps including homogenization, phase separation, RNA precipitation, RNA wash and RNA re-dissolving. The final RNA solution was stored at $-80^{\circ} \mathrm{C}$. Following quality control tests of the extracted RNA, miRNAs were subsequently labeled with fluorescent dyes. Samples containing labeled miRNAs were then hybridized with miRCURY LNA ${ }^{\mathrm{TM}}$ microRNA Array (v.18.0, Exiqon). The images were scanned and analyzed. Abnormally expressed miRNAs found through microarray analysis were further validated by real-time quantitative PCR using plasma samples from patient subjects. All tissue samples and postoperative plasma samples were subjected to real-time quantitative PCR in order to verify whether the above abnormally expressed miRNAs were also abnormally expressed in these samples.

Statistical method. Statistical analysis was performed using the SPSS 18.0 software. Measurement data were expressed as mean \pm standard deviation, and the t-test was used for comparison between groups. Count data were expressed as percentage, and the Chi-square test was used for comparison between groups. ROC curve was used to evaluate diagnostic powers of sonographic TI-RADS classification, circulating miRNA expression profiling, and their combination for malignant thyroid nodules. Sensitivity $=$ number of true-positive $/$ (number of true-positive + number of false-negative) x $100 \%$. Specificity $=$ number of true-negative $/$ (number of true negative + number of false-positive) $\times 100 \%$. A difference was statistically significant at $\mathrm{P}<0.05$.

\section{Results}

Comparison of sonographic TI-RADS classification and pathological diagnosis. Of the 212 nodules, 2 fell into TI-RADS category 2 and were diagnosed as benign. Malignant nodules accounted for $4.35,37.14,84.78,93.33$ and $96.77 \%$ of the nodules that fell into TI-RADS categories 3, 4a, 4b, 4c and 5, respectively. A nodule that fell into a higher TI-RADS classification category had a higher risk of malignancy. Of the 121 patients, $92.55 \%$ had nodular goiter, $3.31 \%$ had inflammatory nodules, $2.48 \%$ toxic nodular goiter, $0.83 \%$ thyroid cysts and $0.83 \%$ thyroid tumors (Tables I and II). 
Table I. Comparison of sonographic TI-RADS classification and pathological diagnosis.

\begin{tabular}{|c|c|c|c|c|}
\hline \multirow[b]{2}{*}{ TI-RADS category } & \multicolumn{2}{|c|}{ Pathological diagnosis } & \multirow[b]{2}{*}{ Case number } & \multirow[b]{2}{*}{ Percentage of malignancy (\%) } \\
\hline & Benign & Malignant & & \\
\hline 2 & 2 & 0 & 2 & 0.00 \\
\hline 3 & 22 & 1 & 23 & 4.35 \\
\hline $4 a$ & 22 & 13 & 35 & 37.14 \\
\hline $4 b$ & 7 & 39 & 46 & 84.78 \\
\hline $4 \mathrm{c}$ & 5 & 70 & 75 & 93.33 \\
\hline 5 & 1 & 30 & 31 & 96.77 \\
\hline Total & 59 & 153 & 212 & 72.17 \\
\hline
\end{tabular}

Table II. Pathological diagnosis.

\begin{tabular}{lrc}
\hline Pathological type & Case (n) & Percentage (\%) \\
\hline Nodular goiter & 112 & 92.55 \\
Inflammatory nodules & 4 & 3.31 \\
Toxic nodular goiter & 3 & 2.48 \\
Thyroid cyst & 1 & 0.83 \\
Thyroid tumor & 1 & 0.83 \\
\hline
\end{tabular}

Diagnostic power of sonographic TI-RADS classification for malignant thyroid nodules. As shown in Table III, the sensitivity, specificity, positive predictive value and negative predictive value of sonographic TI-RADS classification were $90.84,77.97,91.44$ and $76.67 \%$, respectively, in the diagnosis of thyroid nodule malignancy.

Circulating miRNA expression profiling. As shown in Table IV, the expression levels of miRNA146b, miRNA187, miRNA375, miRNA-222-3p and miRNA-151a-5p were higher, while the level of miRNA138 was lower, in patients with either benign (benign group) or malignant (malignant group) thyroid nodules than those in the control group. The expression levels of miRNA146b, miRNA187, miRNA375, miRNA-222-3p and miRNA-151a-5p were higher, while the level of miRNA138 was lower, in patients in the malignant group than the in patients in the benign group. All the differences were statistically significant $(\mathrm{P}<0.05)$.

Diagnostic power of circulating miRNA expression profiling for malignant thyroid nodules. As shown in Table V, the sensitivity, specificity, positive predictive value and negative predictive value of circulating miRNA expression profiling were $89.04,66.67,85.52$ and $73.33 \%$, respectively, in the diagnosis of thyroid nodule malignancy.

ROC curve. Circulating miRNA expression profiling demonstrated its diagnostic value with an AUC of 0.926, area standard error of 0.024 and $95 \%$ confidence interval of $0.880-0.973$, while sonographic TI-RADS classification demonstrated its diagnostic value with an AUC of 0.869, area standard error of 0.027 and $95 \%$ confidence interval of $0.816-0.921$. A ROC

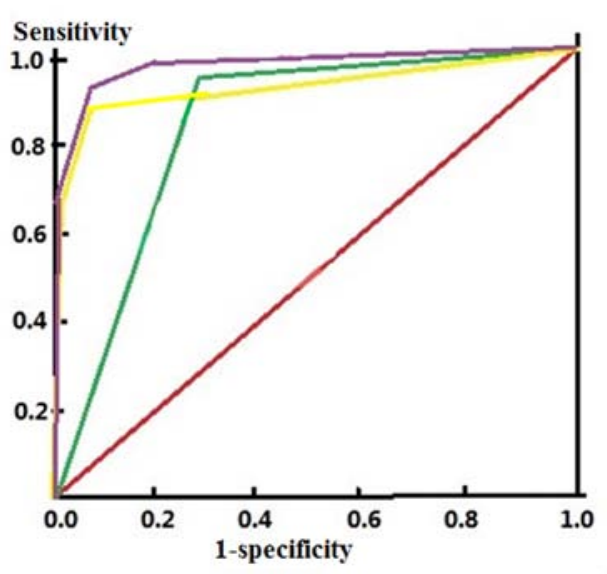

Figure 1. ROC curve showing diagnostic power of sonographic TI-RADS classification, circulating miRNA expression profiling and their combination for malignant thyroid nodules. Red line, the reference line; green line, TI-RADS; yellow line, the circulating miRNA; purple line, the combination of the circulating miRNA and TI-RADS.

curve was established using data of the two diagnostic tools (Figs. 1-3). The AUC of the combined diagnostic method was 0.973 , which was significantly different from the AUC of the individual diagnostic method $(\mathrm{P}<0.05)$.

\section{Discussion}

Thyroid nodules are common signs and symptoms in thyroid diseases. It was reported that the incidence of thyroid nodules is $\sim 3-7 \%$ by palpation alone, whereas the incidence ranges from 20 to $76 \%$ by high-resolution ultrasonography, and $\sim 5-10 \%$ are malignant nodules (7). Ultrasonography is widely used in the diagnosis or differential diagnosis of thyroid nodules due to its simplicity, non-invasiveness and high resolution. However, the morphological manifestations obtained from ultrasonography cannot provide the biological information at genetic and molecular levels required for precision medicine, and cannot provide the information needed for targeted therapy and personalized treatment either (8). Given the results of previous studies, a combination of molecular biology and ultrasonography was explored in this study. miRNA is a small non-coding single-stranded RNA molecule containing 22 nucleotides. It is formed in eukaryotes through genome encoding, transcription 
Table III. Diagnostic power of sonographic TI-RADS classification for malignant thyroid nodules.

\begin{tabular}{lccccccc}
\hline & \multicolumn{2}{l}{ Pathological diagnosis } & & & & \\
\cline { 2 - 5 } $\begin{array}{l}\text { TI-RADS } \\
\text { diagnosis }\end{array}$ & Malignant & Benign & Total & $\begin{array}{c}\text { Sensitivity } \\
(\%)\end{array}$ & $\begin{array}{c}\text { Specificity } \\
(\%)\end{array}$ & $\begin{array}{c}\text { Positive predictive } \\
\text { value }(\%)\end{array}$ & $\begin{array}{c}\text { Negative predictive } \\
\text { value }(\%)\end{array}$ \\
\hline Malignant & 139 & 13 & 152 & 90.84 & 77.97 & 91.44 & 76.67 \\
Benign & 14 & 46 & 60 & & & & \\
Total & 153 & 59 & 212 & & & \\
\hline
\end{tabular}

Table IV. Circulating miRNA expression profiling.

\begin{tabular}{lccccc}
\hline miRNA & Benign group $(\mathrm{n}=59)$ & Malignant group $(\mathrm{n}=253)$ & Control group $(\mathrm{n}=212)$ & $\mathrm{F}$ & P-value \\
\hline miRNA146b & $5.87 \pm 1.18$ & $9.97 \pm 2.21$ & $2.24 \pm 1.07$ & 6.921 & 0.021 \\
miRNA187 & $22.24 \pm 1.07$ & $33.07 \pm 11.16$ & $6.56 \pm 1.72$ & 4.997 & 0.040 \\
miRNA138 & $6.62 \pm 1.33$ & $3.76 \pm 0.17$ & $8.87 \pm 0.10$ & 8.353 & 0.008 \\
miRNA375 & $12.11 \pm 1.89$ & $31.16 \pm 7.76$ & $3.76 \pm 2.31$ & 4.273 & 0.047 \\
miRNA-222-3p & $21.46 \pm 1.94$ & $28.81 \pm 2.07$ & $15.69 \pm 2.01$ & 8.531 & 0.006 \\
miRNA-151a-5p & $8.56 \pm 1.25$ & $14.94 \pm 3.95$ & $5.22 \pm 0.09$ & & \\
\hline
\end{tabular}

Table V. Diagnostic power of circulating miRNA expression profiling for malignant thyroid nodules.

\begin{tabular}{lccccccc}
\hline & \multicolumn{2}{l}{ Pathological diagnosis } & & & & \\
\cline { 2 - 6 } $\begin{array}{l}\text { Diagnostic } \\
\text { results }\end{array}$ & Malignant & Benign & Total & $\begin{array}{c}\text { Sensitivity } \\
(\%)\end{array}$ & $\begin{array}{c}\text { Specificity } \\
(\%)\end{array}$ & $\begin{array}{c}\text { Positive predictive } \\
\text { value }(\%)\end{array}$ & $\begin{array}{c}\text { Negative predictive } \\
\text { value }(\%)\end{array}$ \\
\hline Malignant & 130 & 22 & 152 & 89.04 & 66.67 & 85.52 & 73.33 \\
Benign & 16 & 44 & 60 & & & & \\
Total & 146 & 66 & 212 & & & & \\
\hline
\end{tabular}

and cleavage. miRNA acts as post-transcriptional regulator of its target gene (9). Clinical studies on association of miRNAs with a series of cancers such as colon cancer, gastric cancer, lung cancer, liver cancer, esophageal cancer, bladder cancer, and cervical cancer showed that miRNAs not only can maintain stability of the physiological environment, but also can promote formation of a vicious circle of the disease (10). This study was aimed to increase the diagnostic accuracy for malignant thyroid nodules by combining sonographic TI-RADS classification and circulating miRNA expression profiling. The combined diagnostic method can reduce unnecessary puncture and surgery for patients.

Some scholars in China have studied circulating miRNAs in prostate cancer. Mitchell et al (11) transplanted prostate cancer cells in mice, and then found tumor-derived miRNAs in their plasma, high expression of miR-100, miR-141, miR-125b, miR-205, miR-143, miR-296, in prostate cancer tissues, as well as significantly elevated contents in serum. Therefore, this study selected miRNA146b, miRNA187, miRNA375, miRNA-222-3p, miRNA-151a-5p and miRNA138 that have been studied in the clinic. Normally, a two-dimensional sonogram of a patient with thyroid cancer shows low-level echoes, irregular margins and unclear boundaries. If a sonogram shows a single low-level echo and a blurred boundary at the left thyroid inferior pole, the pathological result often points to papillary thyroid carcinoma. In such cases, calcification is often observed inside the thyroid nodules (12). In clinic, evaluation of nodule boundary can be used to help differentiate benign and malignant thyroid nodules. Malignant thyroid nodules often present a blurred boundary compared to benign nodules, and the marginal irregularity rate is characteristically high in thyroid cancer patients (13). Sonographic TI-RADS classification is a comprehensive interpretation of the sonograms of thyroid nodules. It is practical to obtain the sonogram using routine ultrasound equipment and categorize the information using TI-RADS. Therefore, this diagnostic method can be promoted easily in community hospitals (14). In this study, of the 212 nodules, 2 fell into TI-RADS category 2 and were diagnosed as benign. Malignant nodules accounted for $4.35,37.14,84.78,93.33$ and $96.77 \%$ of the nodules fell into TI-RADS categories 3, 4a, 4b, 4c and 5, respectively. A nodule 


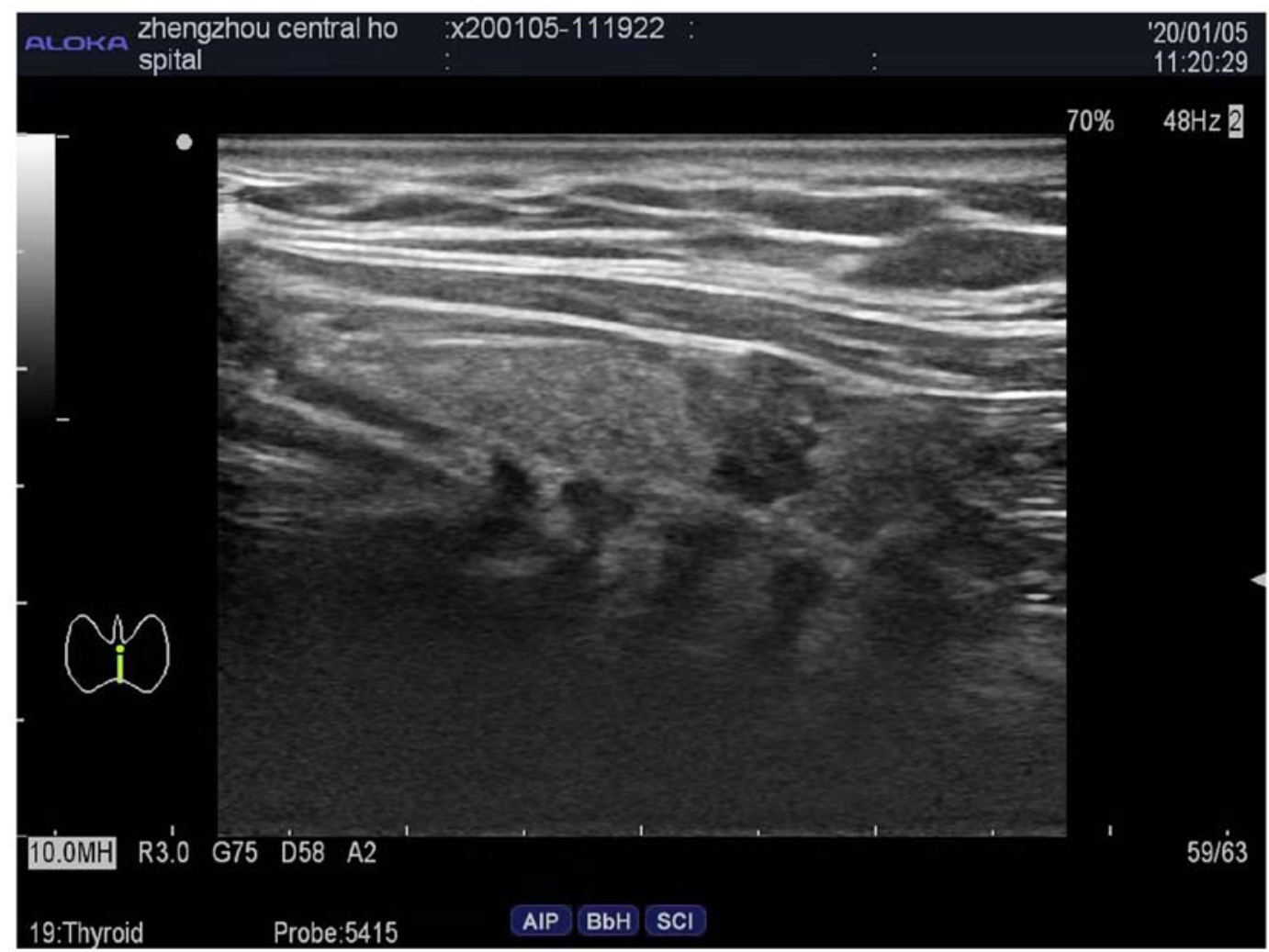

Figure 2. Nodular goiter. A two-dimensional grayscale map TI-RADS 3.

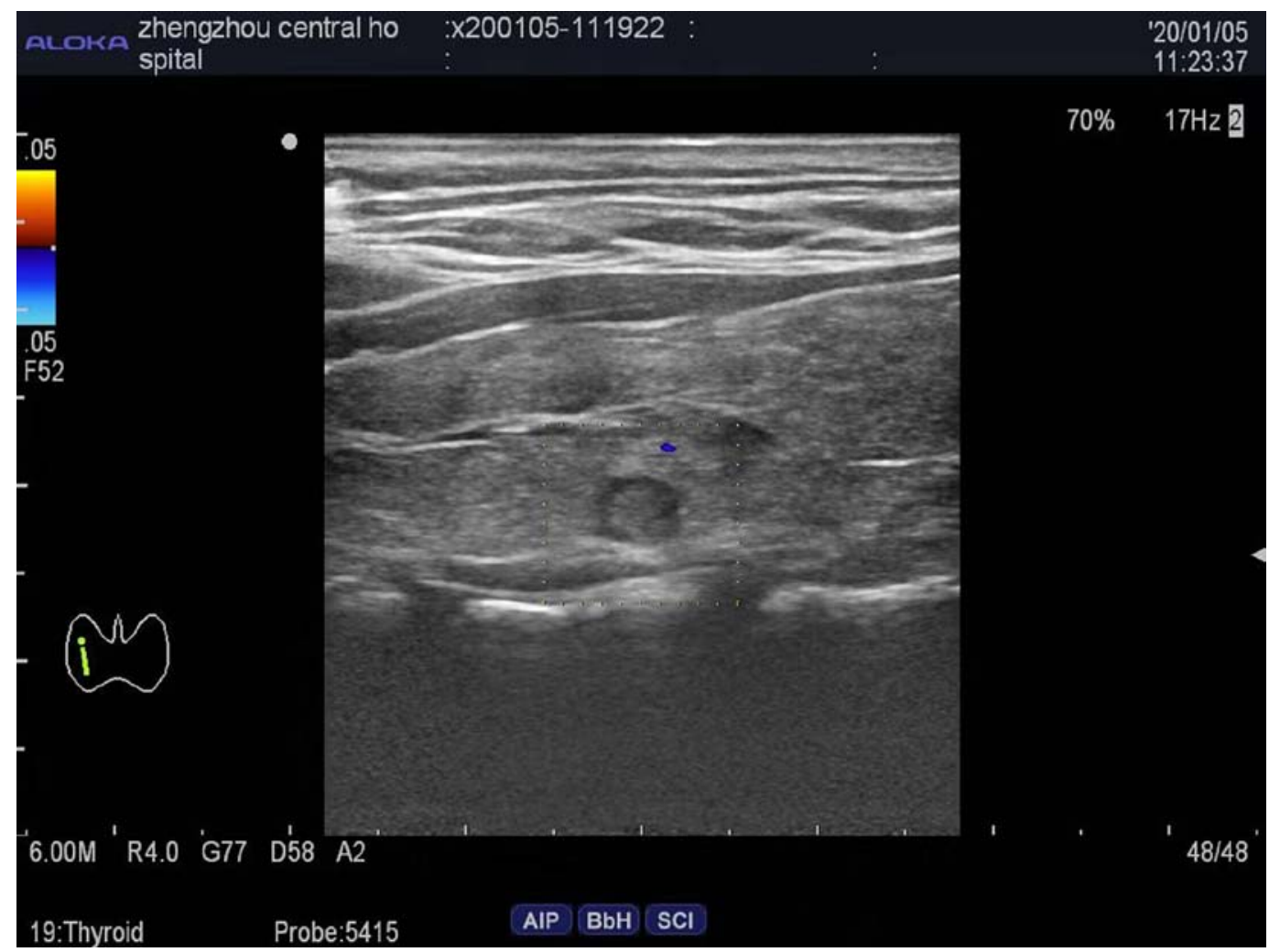

Figure 3. Papillary carcinoma. A two-dimensional grayscale map TI-RADS 4b.

that fell into a higher TI-RADS classification category had a higher risk of malignancy. Of the 121 patients, $92.55 \%$ cases had nodular goiter, $3.31 \%$ cases had inflammatory nodules,
$2.48 \%$ cases had toxic nodular goiter, $0.83 \%$ cases had thyroid cysts and $0.83 \%$ cases had thyroid tumors. The sensitivity, specificity, positive predictive value and negative 
predictive value of sonographic TI-RADS classification were $90.84,77.97,91.44$ and $76.67 \%$, respectively, in the diagnosis of thyroid nodule malignancy. The findings demonstrated that sonographic TI-RADS classification has excellent diagnostic power. However, this diagnostic method raises the bar high for sonographers' professional credentials. Diagnostic sonographers need to be skilled in the operation of ultrasound equipment, especially the method to distinguish visual acuity of abnormal structures, and be able to observe and evaluate the abnormal structures from multiple angles and multiple sections. miRNAs are important modulators of inflammatory responses and cancers. Through miRNAs, cancers can trigger inflammatory responses, which in turn modulate miRNA gene expression and induce carcinogenesis (15). A single miRNA molecule can bind to 3' untranslated regions (3'-UTRs) of the target gene mRNA in incompletely complementary manner and thereby inhibits target gene translation. At the same time, it modulates gene expression in multiple pathways. When carcinogenesis occurs, the patient's immune system is unbalanced and tends to promote the process of carcinogenesis. At this time, a non-self-limited cancer-related inflammatory response is triggered, resulting in abnormal miRNA expression (16). In this study, the expression levels of miRNA146b, miRNA187, miRNA375, miRNA-222-3p and miRNA-151a-5p were higher, while the level of miRNA138 was lower, in patients with either benign (benign group) or malignant (malignant group) thyroid nodules than those in the control group. The expression levels of miRNA146b, miRNA187, miRNA375, miRNA-222-3p and miRNA-151a-5p were higher, while the level of miRNA138 was lower, in patients in malignant group than those in patients in benign group. All the differences were statistically significant $(\mathrm{P}<0.05)$. Both miRNA146b and miRNA187 are common biomarkers for the early diagnosis of gastric cancer in clinical practice. They are key regulatory miRNAs that are associated with cancer and inflammation, acting as indicators of the presence of oncogenes. As biomarkers for diagnosis and prognostic prediction of various cancers, these two miRNAs are of clinical significance. It has been reported (17) that miRNA-222-3p and miRNA-151a-5p are highly expressed in patients with papillary thyroid carcinoma, tumor infiltration, medullary carcinoma, tumor diameter $>4 \mathrm{~cm}$ and distant metastatic tumors; as cancer-causing molecules, they can promote cell proliferation by inhibiting a tumor suppressor gene. According to a previous study (18), miR-138 overexpression is a negative regulator of mesangial cell cytoprotective factor SIRT1, which inhibits nitric oxide production through endothelial nitric oxide synthase, thus negative regulation of miR-138 could inhibit the fibrosis and inflammatory response during glomerular mesangial damage, suggesting that negative regulation of miR-138 could inhibit thyroid disease. Moreover, the expression of miR-375 in gastric cancer tissues is significantly downregulated, probably due to the negative regulation of the transcription factor Snail, which is upregulated in gastric cancer, suggesting that miR-375 may be involved in the development of gastric cancer (19). Overexpression of miR-375 or decreased expression of Jak2 can significantly inhibit the proliferation, migration and invasion of gastric cancer cells. The inhibition of miR-375 overexpression can be reversed by its possible downstream target Jak2, suggesting that miR-375 be involved in the development of gastric cancer by regulating the expression of Jak2 (20). The results of this study showed that the sensitivity, specificity, positive predictive value, and negative predictive value of circulating miRNA for the diagnosis of malignant thyroid nodules were 89.04, 66.67, 85.52 and $73.33 \%$, respectively. This suggests that circulating miRNAs have a good diagnostic effect on thyroid nodules. The combination of TI-RADS ultrasound classification and circulating miRNA gene detection for the diagnosis of thyroid nodules showed that the diagnostic effect was better than that of the two methods alone. This may be due to the fact that circulating miRNA gene detection can meet the biological information at the genetic and molecular levels required for precision medicine, and meet the information needed for targeted therapy and personalized organisms, thereby improving the diagnostic effect. In this study, it was found that TI-RADS combined with circulating miRNA did not change the category of nodules, and only showed better sensitivity and specificity in the diagnosis of thyroid nodules. However, circulating miRNA will increase the economic burden. In clinical practice, patients should choose whether to check with circulating miRNA according to their own conditions. Other methods can be used to detect and diagnose thyroid nodules. TI-RADS combined with circulating miRNA diagnosis can be used in cases where clinical diagnosis is difficult.

In conclusion, sonographic TI-RADS classification combined with circulating miRNA expression profiling can improve the diagnosis of thyroid nodules.

\section{Acknowledgements}

Not applicable.

\section{Funding}

No funding was received.

\section{Availability of data and materials}

The datasets used and/or analyzed during the present study are available from the corresponding author on reasonable request.

\section{Authors' contributions}

YM wrote the manuscript FZ, LH and ZK led the conception and design of this study. YM, FZ, FL, LL and YH were responsible for the data collection and analysis. YM and ZK were in charge of interpreting the data and drafting the manuscript. YM and FZ helped with statistical analysis. The final version was read and accepted by all the authors.

\section{Ethics approval and consent to participate}

The study was approved by the Ethics Committee of Zhengzhou Central Hospital Affiliated to Zhengzhou University (Zhengzhou, China). Signed informed consents were obtained from the patients and/or the guardians.

\section{Patient consent for publication}

Not applicable. 


\section{Competing interests}

The authors declare that they have no competing interests.

\section{References}

1. Liu BX, Xie XY, Liang JY, Zheng YL, Huang GL, Zhou LY, Wang $\mathrm{Z}, \mathrm{Xu} \mathrm{M}$ and Lu MD: Shear wave elastography versus real-time elastography on evaluation thyroid nodules: A preliminary study. Eur J Radiol 83: 1135-1143, 2014

2. Sun J, Cai J and Wang X: Real-time ultrasound elastography for differentiation of benign and malignant thyroid nodules: A meta-analysis. J Ultrasound Med 33: 495-502, 2014.

3. Chen KY, Chen CN, Wu MH, Ho MC, Tai HC, Kuo WH, Huang WC, Wang YH, Chen A and Chang KJ: Computerized quantification of ultrasonic heterogeneity in thyroid nodules. Ultrasound Med Biol 40: 2581-2589, 2014.

4. Hoang JK, Middleton WD, Farjat AE, Langer JE, Reading CC Teefey SA, Abinanti N, Boschini FJ, Bronner AJ, Dahiya N, et al: Reduction in thyroid nodule biopsies and improved accuracy with American College of Radiology Thyroid Imaging Reporting and Data System. Radiology 287: 185-193, 2018.

5. Coskun E, Neumann M, Schlee C, Liebertz F, Heesch S, Goekbuget N, Hoelzer D and Baldus CD: MicroRNA profiling reveals aberrant microRNA expression in adult ETP-ALL and functional studies implicate a role for miR-222 in acute leukemia. Leukemia 37: 647-656, 2013.

6. Kwak JY, Han KH, Yoon JH, Moon HJ, Son EJ, Park SH, Jung HK, Choi JS, Kim BM and Kim EK: Thyroid imaging reporting and data system for US features of nodules: a step in establishing better stratification of cancer risk. Radiology 260: 892-899, 2011.

7. Cakal E, Sahin M, Ünsal İÖ, Güngünes A, Akkaymak E, Ôzkaya EÇ, Bozkurt NÇ, Özbek M and Delibaşı T: Elastography in the differential diagnosis of thyroid nodules. Ultrason Imaging 37: 251-257, 2015.

8. Zhang XL and Qian LX: Ultrasonic features of papillary thyroid microcarcinoma and non-microcarcinoma. Exp Ther Med 8: 1335-1339, 2014.

9. Zhu M, Yi M, Kim CH, Deng C, Li Y, Medina D, Stephens RM and Green JE: Integrated miRNA and mRNA expression profiling of mouse mammary tumor models identifies miRNA signatures associated with mammary tumor lineage. Genome Biol 12: R77, 2011.

10. Lerebours F, Cizeron-Clairac G, Susini A, Vacher S, Mouret-Fourme E, Belichard C, Brain E, Alberini JL, Spyratos F, Lidereau $\mathrm{R}$, et al: miRNA expression profiling of inflammatory breast cancer identifies a 5-miRNA signature predictive of breast tumor aggressiveness. Int J Cancer 133: 1614-1623, 2013.
11. Mitchell PS, Parkin RK, Kroh EM, Fritz BR, Wyman SK, Pogosova-Agadjanyan EL, Peterson A, Noteboom J, O'Briant KC, Allen A, et al: Circulating microRNAs as stable blood-based markers for cancer detection. Proc Natl Acad Sci USA 105: 10513-10518, 2008.

12. Riazi A, Kalantarhormozi M, Nabipour I, Eghbali SS, Farzaneh M, Javadi H, Ostovar A, Seyedabadi M and Assadi M: Technetium-99m methoxyisobutyl isonitrile scintigraphy in the assessment of cold thyroid nodules: Is it time to change the approach to the management of cold thyroid nodules? Nucl Med Commun 35: 51-57, 2014.

13. Dogan S, Atmaca A, Dagdelen S, Erbas B and Erbas T: Evaluation of thyroid diseases and differentiated thyroid cancer in acromegalic patients. Endocrine 45: 114-121, 2014.

14. Basha MAA, Alnaggar AA, Refaat R, El-Maghraby AM, Refaat MM, Abd Elhamed ME, Abdalla AAEM, Aly SA, Hanafy AS, Mohamed AEM, et al: The validity and reproducibility of the thyroid imaging reporting and data system (TI-RADS) in categorization of thyroid nodules: Multicentre prospective study. Eur J Radiol 117: 184-192, 2019.

15. LabourierE, Shifrin A,Busseniers AE,Lupo MA,Manganelli ML, Andruss B, Wylie D and Beaudenon-Huibregtse S: Molecular testing for miRNA, mRNA, and DNA on fine-needle aspiration improves the preoperative diagnosis of thyroid nodules with indeterminate cytology. J Clin Endocrinol Metab 100: 2743-2750, 2015.

16. Rossing M, Kaczkowski B, Futoma-Kazmierczak E, Glud M, Klausen M, Faber J, Nygaard B, Kiss K, Sørensen CH, Nielsen FC, et al: A simple procedure for routine RNA extraction and miRNA array analyses from a single thyroid in vivo fine needle aspirate. Scand J Clin Lab Invest 70: 529-534, 2010.

17. Ratert N, Meyer HA, Jung M, Lioudmer P, Mollenkopf HJ, Wagner I, Miller K, Kilic E, Erbersdobler A, Weikert S, et al: miRNA profiling identifies candidate miRNAs for bladder cancer diagnosis and clinical outcome. J Mol Diagn 15: 695-705, 2013.

18. Ma Y, Liu M, Tan T, Yan A, Guo L, Jiang K, Tan C and Wan Y: Deep eutectic solvents used as extraction solvent for the determination of flavonoids from Camellia oleifera flowers by high-performance liquid chromatography. Phytochem Anal 29: 639-648, 2018.

19. Zhou SY, Wang R, Deng LQ, Zhang XL and Chen M: A new isoflavanone from Ficus tikoua Bur. Nat Prod Res 32: 2516-2522, 2018.

20. Croce CM: Causes and consequences of microRNA dysregulation in cancer. Nat Rev Genet 10: 704-714, 2009.

This work is licensed under a Creative Commons Attribution-NonCommercial-NoDerivatives 4.0 International (CC BY-NC-ND 4.0) License. 